\title{
Comparative study of Ultrasonography with Fine Needle Aspiration Cytology in the diagnosis of causes of Obstructive Jaundice.
}

\section{Pandit SP1 , Pandit $S^{2}$, Pandit S}

${ }^{1}$ Chief Consultant Radiologist, Bir Hospital, Kathmandu, ${ }^{2}$ Medical officer, Bir hospital, Kathmandu, ${ }^{3}$ Resident, Internal Medicine, Presence Saint Hospital, Chicago, USA.

Correspondence to: Prof Dr Swoyam Prakash Pandit; Email- $\underline{\text { swoyampandit@ hotmail.com }}$

\begin{abstract}
Introduction: Surgical jaundice or obstructive jaundice is one of the common causes of jaundice. It is important cause of morbidity and mortality in an adult, common at 40-60 years of age and the incidence is increasing day by day. The purpose of this study was to use easily available ultrasonography in the diagnosis of causes of obstructive jaundice and to determine its effectiveness by comparing with Fine Needle Aspiration Cytology (FNAC).
\end{abstract}

Methods: Non- randomized, prospective study of 35 cases with clinical features of jaundice and altered bio-chemical test was conducted over a period of one year. The ultrasound findings were compared with FNAC to characterize the cause of obstruction.

Result: Out of 35 cases, 24 cases were subjected to USG guided FNAC for suspicion of malignancy for confirmation. USG showed 18(51\%) had benign causes, 11(31\%) malignant and $6(17 \%)$ were indeterminate. FNAC result showed $13(54 \%)$ were malignant. Over all accuracy of USG in determination of cause of obstruction is high as $83 \%$. The efficacy of the USG is acceptable when compared with histopathology ( $\mathrm{P}$ value is 0.6816 ).

Conclusion: Ultrasonography correctly predicted the causes of obstruction whereas in malignant cases, when combined with other modality, accuracy of diagnosis can be increased.

Keywords: Bilirubin; Fine Needle Aspiration Cytology; Obstructive jaundice; Ultrasonography. 


\section{INTRODUCTION}

Jaundice is a clinical condition in which the body fluids and tissues, particularly eyes, skin takes yellowish color as a result of excess bilirubin ${ }^{1}$. Some natives living in high altitude and natives of China, Japan, Korea, South American ancestry have greater prevalence of elevated bilirubin in healthy population ${ }^{2}$. The incidence of Jaundice is increasing rapidly. The most common cause being neoplastic, calculus diseases and stricture.

The diagnostic capacity of USG has increased in recent years. In addition USG is used not only for diagnostic tool but also to enhance safety and effectiveness of other diagnostic tools as well as therapeutic potentials ${ }^{3}$. The availability of adequate services and the cost of the USG have proven its benefit to the jaundice patient. The modality is widely available in most of the hospitals and treatment centers in our country.

Tissue diagnosis is important in biliary obstructive patients. FNAC is the tool for accurate diagnosis of malignancy in suspicious or equivocal cases.

\section{METHODS}

Non randomized, prospective study was carried out in the Department of Radio diagnosis and Imaging in the tertiary care center in Kathmandu, Nepal over a period of one year. The subjects had features of obstructive jaundice with written consent were included. Patients who were unfit for invasive investigation and patient unwilling to participate in study were excluded. Transabdominal ultrasonogram was obtained in all 35 cases with commercially available real time scanner with color Doppler (Apllio 400 Toshiba) Ultrasound machine with transducer frequency of 3.5 MHZ convex probe after 4 hours of fasting. Scanning was done in oblique, transverse and sagittal views. Coronal imaging was performed from anterior axillary line with gallbladder as acoustic window for distal bile duct pathology. Measurements were made using electronic caliper and images were recorded in thermal printer.

The scan was assessed for biliary dilatation, causes of obstruction, focal or diffuse thickening of bile ducts, hepatic tumors, lymphadenopathy, portal vein thrombosis \& cholelithiasis. The dilated IHBDS and extrahepatic biliary tree stricture were evaluated by USG and recorded. The cause of obstruction (neoplastic, benign, and indeterminant) was mentioned during scanning. Ultrasonography guided FNAC in suspicious cases was performed for tissue diagnosis.

The numerical data obtained from the study was compiled and analyzed, using standard statistical calculation. $\mathrm{P}$ value $<0.05$ was considered to be statistically significant. "P" value was calculated using chi-square test.

\section{RESULT}

Out of total 35 cases, $19(54.5 \%)$ were male and 16(45.5\%) were female and age ranged from 20 to 81 year (mean age 51 year). All (100\%) patient presented with clinical features of jaundice and $18(51 \%)$ 
cases presented as pain abdomen as chief complaint. (Table 1)

Table1. Distribution of patients according to sign and symptoms.

\begin{tabular}{|l|c|}
\hline \multicolumn{1}{|c|}{ Sign and Symptom } & Total (\%) \\
\hline Pain abdomen & $18(51 \%)$ \\
\hline Jaundice & $35(100 \%)$ \\
\hline Weight loss & $15(43 \%)$ \\
\hline Other( itching, clay stool) & $14(40 \%)$ \\
\hline
\end{tabular}

After clinical assessment all cases were subjected to USG. Of the total 35 cases, USG showed $11(31 \%)$ cases malignant, $18(51 \%)$ benign causes, and $6(17 \%)$ indeterminate. (Table 2)

Table2. Distribution of patients according to cause of obstruction as shown by USG.

\begin{tabular}{|l|c|}
\hline \multicolumn{1}{|c|}{ Cause } & Total (\%) \\
\hline Benign & $18(51 \%)$ \\
\hline Malignancy & $11(31 \%)$ \\
\hline Indeterminate & $6(17 \%)$ \\
\hline Total & $\mathbf{3 5}$ \\
\hline
\end{tabular}

USG guided FNAC was done in 24 cases of suspected Malignancy for confirmation. The iatrogenic stricture of CBD was seen in 11 patients who had previous history of surgery. Out of 24 FNAC cases, 13 were confirmed to have malignancy, 1 case to have benign cause and 10 were indeterminate. (Table 3)

Table3. Distribution of patients according to cause of obstruction as show by FNAC.

\begin{tabular}{|l|c|}
\hline \multicolumn{1}{|c|}{ Cause } & Total (\%) \\
\hline Benign & $1(3 \%)$ \\
\hline Malignancy & $13(54 \%)$ \\
\hline Indeterminate & $10(42 \%)$ \\
\hline Total & $\mathbf{2 4}$ \\
\hline
\end{tabular}

The efficacy of the USG is acceptable when compared with histopathology and assessed by statistical calculation applying chi-square test is 0.26 and $P$ value is 0.6816 .

\section{DISCUSSION}

This study showed the evaluation of predictive value of USG in the diagnosis of cause of obstructive jaundice. They were matched with FNAC result. Besides USG many other modalities such as CT, PTC, ERCP, Radionuclide imaging, MRI/MRCP etc will help in the diagnosis. Among them, USG is easily available and is the first choice of modality to locate the possible cause in common practice.

In this study, the incidence of neoplastic lesion is high in 40-60 yrs and benign lesion (stone, postoperative stricture, choledochal cyst) high in age below 40 $\mathrm{yrs}^{4}$. Malignancy was the most common cause of obstruction of biliary tree ${ }^{5}$ and benign is less common and few were indeterminate. In case of Gall bladder cancer, the incidence of cholelithiasis is 70-90\%, Porcelain or calcified Gall bladder can be precursor of cancer. Biochemical test should be performed in all suspected cases of obstruction. When isolated level of alkaline phosphate level is elevated, even in absences of evidence of 
liver dysfunction this is an indication of biliary obstruction and needs further investigation ${ }^{2}$.

Raised bilirubin level alone is insensitive test. The normal range of bilirubin is 0.2 $1.0 \mathrm{mg} / \mathrm{dl}$, alkaline phosphate is $50-80$ $\mathrm{mg} / \mathrm{dl}$. The two or three fold rise of alkaline phosphate level in blood strongly suggests biliary obstruction.

The Gall Bladder cancer is an aggressive disease with overall survival rate of about $5 \%$ and median survival rate of 6 month $^{6}$.

The presence of layered bile or homogeneously echogenic bile in dependent layer without distal acoustic shadows is biliary sludge. It is commonly seen in extra hepatic biliary obstruction. Similarly USG is a sensitive tool to differentiate medical from surgical jaundice, when laboratory and clinical assessment are in doubt. Dilated intra hepatic biliary radicals (normal $1-3 \mathrm{~mm}$ ) and dilated common bile duct (normal $8 \mathrm{~mm})$ are identified by $\mathrm{USG}^{6}$.

The accuracy of USG to diagnose dilated biliary tree is $97 \%$. The dilated biliary tree has been grouped into 3 grades:

GRADE I: Central dilatation of IHBD, right or left main hepatic ducts and duct less are dilated $>3 \mathrm{~mm}$.

> GRADE II: Moderate or intermediate dilatation.

GRADE III: Marked dilatation of IHBD, dilated up to the peripheral $1 / 3^{\text {rd }}$ of liver parenchyma.

Similarly, extra hepatic biliary duct is also evaluated by USG. Duct is dilated if diameter is more than $8 \mathrm{~mm}$ in non cholecystectomised and $>10 \mathrm{~mm}$ in post cholecystectomised patient, but distal end of CBD is difficult to visualize in most cases and require Magnetic Resonance Cholangio-Pancreaticography( MRCP) or Endoscopic retrograde cholangiopancreatography (ERCP).

The stricture of common bile duct is also grouped into 3 types by Ultrasound ${ }^{7}$ :

$>$ Type 1 - smooth tapering stenosis with proximal dilatation.

$>$ Type 2 - abrupt cut off of CBD

$>$ Type 3 - Presence of echogenic nodules without acoustic shadows suggestive of stricture and should confirmed by MRCP/ERCP.

In malignant disorder, USG shows echogenic mass with irregular outline or a focal thickening of gall bladder wall with or without obstruction in the CBD and IHBDs. Cholangiocarcinoma is more echogenic than GB fossa mass. Besides this, USG is sensitive to detect secondaries in the liver as hypoechoic mass. Hilar lymphadenopathy, extrinsic compression of the biliary system is also visualized by USG.

In small lesion (calculi, stricture, and tumors) per operative USG is useful to demonstrate these lesions and may be an additional technique in the future. Endoscopic USG has also proved useful in the more detailed assessment of biliary diseases and staging of malignancy. Non operable cases or non-resectable malignant obstruction are managed by external/ internal biliary drainage ${ }^{8}$.

The biliary obstruction is found in $2-10 \%$ of calculus disease, $8 \%$ in Caroli's Disease and $9 \%$ in Sclerosing cholangitis, $1 \%$ in 
Ulcerative colitis, Congenital anomalies of biliary system ${ }^{9}$.The abdominal pain and jaundice are the first symptoms in all patients. USG guided FNAC was performed in all suspected cases of malignancy.

\section{CONCLUSION}

Obstructive jaundice is the commonest complication of biliary malignancy. This study demonstrates that USG with clinical assessment can be utilized as a first line of investigation and in the early diagnosis of malignant lesion. It can also be utilized for FNAC, therapeutic drainage tube as well as stent placement into the biliary ducts in non-resectable patient.

\section{REFERENCES}

1. Sutton D. Textbook of Radiology \& imaging. $\quad 6^{\text {th }}$ ed. Churchill Livingstone ;1998.p962-967.

2. Hegy $T$.et al. The protective role of bilirubin in oxygen radical diseases of the infant. Journal of perinatology 1994 ; 14(4) :269-300.

3. Lain FC, Brooke R, Wing VW et al . Biliary dilation -defining level \& cause by real time Ultrasound 1986; 160: 39-42.

4. Gibson RN, Yenng E, Thompson $\mathrm{NN}$ et al. Bile duct obstruction radiological evaluation of level cause and tumor respectability. Radiology 1986; 160: 43-47.

5. Robledo R, Muro A, Priento ML. Extra hepatic bile duct Carcinoma US characteristic \& accuaracy in demonstration of Tumor. AJR 1996; 198:869-875.

6. Grainger RG, Allision. Diagnostic Radiology. $\quad 3^{\text {rd }}$ ed, Churchill Livingstone Vol II ;1999.p 1214-15.

7. Honickmum SP, Mueller PR Mittenberg et al. Ultrasound in obstructive jaundice-Prospective evaluation of site \& causes. Radiology 1983;147: 511-15.

8. Tio TL, Tutgal G. Endoscopic USG of bile duct \& malignancy, preoperative assessment of local respectability. Gastrointestinal Imaging 1989; 12 :151-157.

9. Berry M, Chaowdhary V, Suri S. Hepatobiliary and gastrointestinal imaging . Jaypee;1997. p70-73. 